\title{
Survival Rates of Patients Treated by Home and Hospital Dialysis and Cadaveric Renal Transplantation
}

\author{
JOHN F. MOORHEAD,* M.B., M.R.C.P. ; R. A. BAILLOD, $\dagger$ M.B., B.S. ; J. P. HOPEWELL, $\ddagger$ F.R.C.s. \\ A. H. KNIGHT, $\$ M.B., M.R.C.P. ; R. E. CROCKETT,\| M.B., B.ch., B.A.o; O. N. FERNANDO,g F.R.C.S. \\ Z. VARGHESE, ${ }^{* *}$ M.SC.
}

\begin{abstract}
Cummary: Of 109 patients admitted to the renal failure $\checkmark$ programme of the Royal Free Hospital $80.7 \%$ were surviving after six years. For patients on home dialysis the four-year rate was $\mathbf{8 6 . 2} \%$, more deaths occurring in women than in men. The short-term survival rate of all patients was high-namely, $96.3 \%$ at six months and $\mathbf{9 4 . 4} \%$ at 12 months. Hence large increases in the rate of acceptance of patients as well as successful transplantation are necessary to balance acceptance and loss from this programme.
\end{abstract}

\section{Introduction}

Regular dialysis and renal transplantation are well-accepted methods in the treatment of patients with terminal renal failure. A recent report (Lewis et al., 1969) of the survival data from 14 United States clinics treating 302 patients on hospital dialysis showed that relatively high survival rates could be achieved $(62 \%$ at five years). The survival rates of patients who have received home dialysis, cadaveric renal transplantation, or hospital dialysis in any single renal failure programme, however, have not previously been reported.

The renal failure programme analysed in this paper started in 1963, home dialysis being introduced in 1964 and cadaveric renal transplantation in 1968 . Though the major emphasis has been on home dialysis, several patients have been treated by regular overnight dialysis in hospital, while others have had changes of treatment. The survival results for combined therapy as well as for home dialysis alone are reported.

\section{Patients}

During the 85-month period ended 30 April, 1970, 109 patients were admitted to the programme. Eighty-nine were admitted for home dialysis training, though the number actually receiving treatment at home at any one time was affected by transplantation or the death of some patients. Patients were admitted to the dialysis programme on the normally accepted clinical criteria for terminal renal failure. Cadaveric renal transplantation was performed on 16 patients; with one exception, they received at least three months' regular dialysis therapy before transplantation.

Selection.-Most patients were admitted from other hospitals after undergoing peritoneal dialysis or dietary therapy for varying periods of time. The referral of patients for haemodialysis may have introduced selection factors-for example, referring physicians may have selected those patients whom they believed had a good chance of being accepted. This tendency was probably corrected by other requests for treatment of patients who were in an advanced state of uraemia. To these external factors, other criteria for

\footnotetext{
* Director, Department of Nephrology.

Consultant Urologist.

Medical Registrar, Department of Nephrology.

Late Medical Registrar, Department of Nephrology.

II Late Medical Registrar, Departme

* Biochemist, Department of Nephrology

Royal Free Hospital, London N.W.3.
}

selection have been added by the receiving hospital; these have been set out in detail elsewhere (Moorhead et al., 1970). Furthermore, the criteria for selection were not applied consistently; in fact, most have been abandoned during the last three years.

\section{Treatment and Method of Analysis}

The aim of treatment has been to provide a dialysis regimen which minimizes fluctuations in plasma biochemistry, is capable of dealing with average metabolic loads and occasional dietary indiscretions, but does not interfere seriously with work or home life. These requirements have been met by thrice-weekly 10-hour overnight home dialyses, using automatic monitors with a short preparation time, and low-volume low-resistance Kiil dialysers. Dietary control has been an important factor in establishing and maintaining control of the uraemic state, and all patients ate a prescribed diet of about 3,500-4,500 calories per day, which was reduced if the patients threatened to put on weight. A well-functioning external shunt or arteriovenous fistula was essential for satisfactory dialysis.

The details of dialysis training, the logistics of home dialysis, and the method of access to the blood stream have been published elsewhere (Baillod et al., 1969; Moorhead et al., 1970).

The life table method of analysis (Cutler and Ederer, 1958) has been applied to all patients admitted up to 30 April, 1970. This method is not sensitive when the number of patients is small. Hence, survival rates were not calculated when the number of patients completing any particular interval fell below 14. Survival times have been measured from the closing date of the study. The survival intervals chosen were six months, 12 months, and then yearly to seven years, the initial six-month period chosen to assess early death rates. Patients who had not completed a treatment interval and who were still alive at the closing date were assessed as having a $50 \%$ chance of completing that interval. All patients were followed-up. The survival tines of those who received transplants have not been dea with separately because of the relatively small number o: operations performed. The survival data were considered ander four headings: (1) all patients admitted for the treatment of chronic renal failure, (2) home dialysis patients alone, (3) the survival of men $v$. women, and (4) the causes of death.

\section{Results}

(1) All Patients Admitted for Treatment of Chronic Renal Failure.-There was little difference between the sixmonth survival rate of $96.3 \%$ (S.E. $1.8 \%$ ) and the 12 -month survival rate of $94.4 \%$ (S.E. $2.2 \%$ ) (see Chart and Table I). Between one and two years the rate declined by $6.3 \%$ to $88.1 \%$ (S.E. $3.2 \%$ ), and there was a similar fall $(4.9 \%$ ) at three years. The explanation for the greater fall between 12 months and three years than between 6 and 12 months is not apparent. The decline in survival rate after three years was 


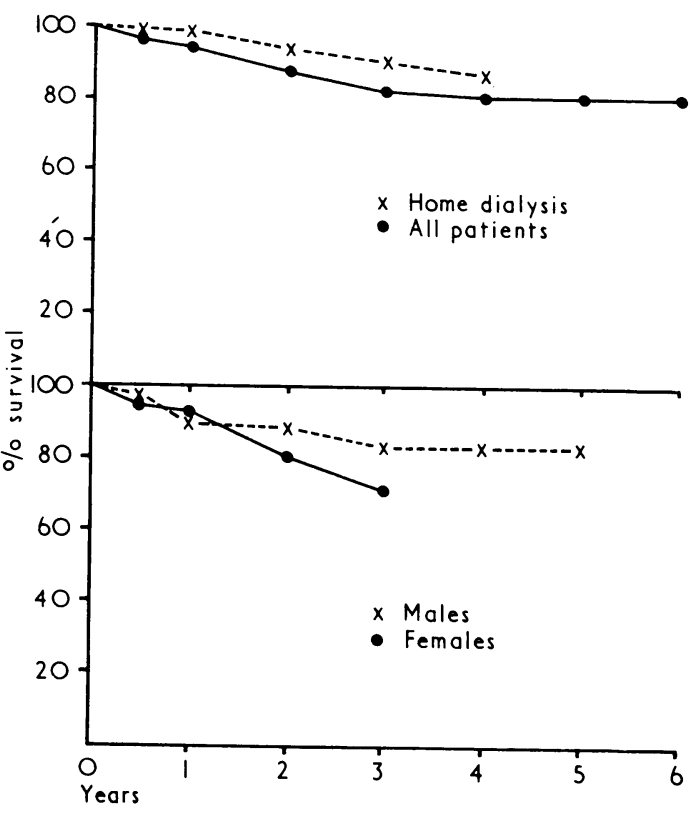

Survival rates.

rather small at $2.5 \%$ to the fourth year, after which no further decline occurred.

(2) Home Dialysis. - The survival times of patients admitted for training for home dialysis were used in computing survival rates for this group, and one patient who died of fulminating hepatitis in hospital at the end of her home training period was therefore included. For those who received transplants the period of independent existence with a functioning transplant was subtracted from the total survival time for each patient. Eleven home dialysis patients received transplants-six were functioning at the close of the study, four failed to start functioning and one was rejected and removed after functioning for a short period. Post transplant

TABle I.-Survival Rates of all Admissions. (No Deaths Occurred in the 3-7 Year Interval)

\begin{tabular}{|c|c|c|c|c|c|c|c|c|}
\hline & \multicolumn{8}{|c|}{ Time after Treatment Started } \\
\hline & \multicolumn{2}{|c|}{ Months } & \multicolumn{6}{|c|}{ Years } \\
\hline & $0-6$ & $7-12$ & 2 & 3 & 4 & 5 & 6 & 7 \\
\hline $\begin{array}{l}\text { No. of patients com- } \\
\text { pleting interval } \ldots \\
\text { Percentage surviving . } \\
\text { Standard error }(\%) . .\end{array}$ & $\begin{array}{r}109 \\
96 \cdot 3 \\
1 \cdot 8\end{array}$ & $\begin{array}{r}101 \\
94 \cdot 4 \\
2 \cdot 2\end{array}$ & $\begin{array}{c}89 \\
88 \cdot 1 \\
3 \cdot 2\end{array}$ & $\begin{array}{c}55 \\
83 \cdot 2 \\
4 \cdot 2\end{array}$ & $\begin{array}{c}33 \\
80 \cdot 7 \\
4 \cdot 9\end{array}$ & $\begin{array}{r}20 \\
80 \cdot 7 \\
4.9\end{array}$ & $\begin{array}{r}14 \\
80 \cdot 7 \\
4.9\end{array}$ & $\frac{3}{-}$ \\
\hline
\end{tabular}

TABLE II.-Survival Rates for Home Dialysis. (No Deaths Occurred in the 4-5 and 5-6 Year Intervals)

\begin{tabular}{|c|c|c|c|c|c|c|c|}
\hline & \multicolumn{7}{|c|}{ Time after Treatment Started } \\
\hline & \multicolumn{2}{|c|}{ Months } & \multicolumn{4}{|c|}{ Years } & \\
\hline & $0-6$ & $7-12$ & 2 & 3 & 4 & 5 & 6 \\
\hline $\begin{array}{l}\text { No. of patients completing } \\
\text { interval } \ldots \\
\text { Percentage surviving } \ldots \\
\text { Standard error }(\%) \\
\text { Sta. }\end{array}$ & $\begin{array}{r}89 \\
98.9 \\
1 \cdot 1\end{array}$ & $\begin{array}{r}82 \\
98 \cdot 9 \\
1 \cdot 1\end{array}$ & $\begin{array}{r}69 \\
93 \cdot 1 \\
3 \cdot 2\end{array}$ & $\begin{array}{r}38 \\
90 \cdot 7 \\
4 \cdot 2\end{array}$ & $\begin{array}{r}20 \\
86 \cdot 2 \\
6 \cdot 6\end{array}$ & $\frac{6}{-}$ & $\underline{1}$ \\
\hline
\end{tabular}

dialysis was not regarded as home dialysis until the patient returned home; and death during the transplant period was not counted as death on home dialysis.

The 6- and 12-month survival rates were identical at $98.9 \%$ (S.E. $1.1 \%$ ) (see Chart and Table II). This group comprised patients who were highly selected in the early years but relatively unselected in the later years of the programme, and it is of considerable interest that the survival rate for this mixed group remained high in the early stages of therapy. Thus selection factors did not seem greatly to influence early survival rates. There was a decline in survival rate of $5.8 \%$ between the first and second years of dialysis therapy, and yearly falls of $2.4 \%$ and $4.5 \%$ to the fourth year, after which the number of patients considered became too small to calculate survival rates. The difference between the one-year survival rate overall and the home dialysis survival rate $(94.4 \%$ v. $98.9 \%$ ) was probably due to the fact that the overall figures included patients with severe complicating disease who died receiving hospital dialysis.

(3) Survival of Males $v$. Females.-In this series twice as many women as men died (Table V), though no single cause of death predominated for either sex. Women appeared to be most at risk in the first two years of treatment, and it is noteworthy that five women died in the second year (Table IV). The three-year survival rate for men was $83.5 \%$ (S.E. $5.3 \%$ ), while for women it was $71.2 \%$ (S.E. $8.9 \%$ ) (see Chart and Table III). The difference is not statistically significant. Six women died while on home dialysis, but no men. The apparent difference between the two survival rates may be due to the small numbers considered.

(4) Causes of Death.-Sixteen patients died. No grouping of the causes of death were noted (Table V). Of the four deaths related to dialysis two were due to hypercalcaemia, one to dialysis disequilibrium, and one to air embolus. Two haemorrhagic states which occurred shortly after dialysis (one cerebral and one gastrointestinal) were possibly related to dialysis heparinization. Two of the deaths due to pulmonary infection (pneumocystis and pseudomonas pneumonia) occurred in transplant patients and were related to immunosuppressive therapy. Two further deaths in home dialysis patients were due to influenzal pneumonia and severe bronchopneumonia, the latter with underlying bronchiectasis. The causes of death confirm that underlying or acquired disease prejudices the survival of patients on haemodialysis. On the other hand, haemodialysis in patients with severe myocardial or immune disease, for example, would be unlikely to affect the fatal course of such diseases.

\section{Discussion}

Though long-term treatment for renal failure has been available for some years, surprisingly few data are available about the survival of patients. Our report is concerned exclusively with survival of patients accepted for the renal failure programme of the Royal Free Hospital. The life table method has been used in computing survival statistics since it offers considerable advantages (Cutler and Ederer, 1958). Thus all survival times contribute to the overall figures, and

TABLE III.-Survival Rates of Males and Females

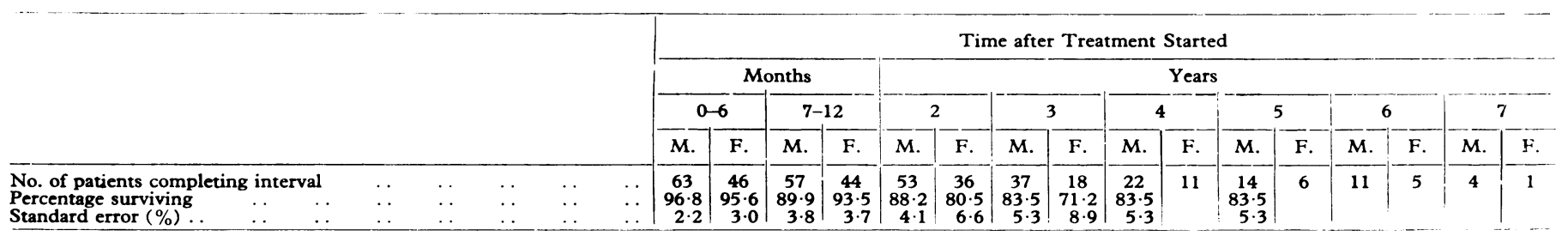

In males no deaths occurred in the 5-6 and 6-7 year intervals.

In males no deaths occurred in the 5-6 and 6-7 year intervals.
In females one death occurred in the 3-4 year interval: Thereafter, no deaths occurred. 


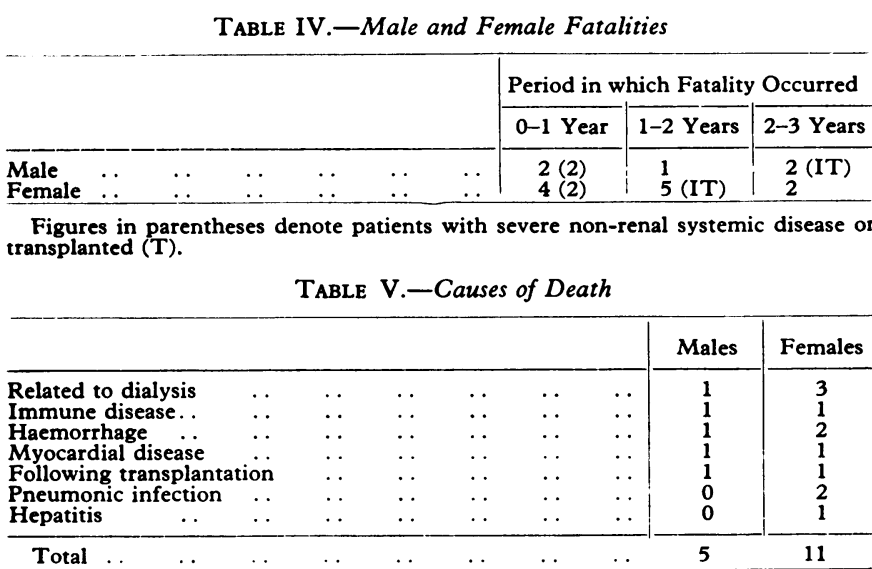

the standard errors applicable to each survival interval can be readily calculated. The calculation of survival rates, however, depends to a great extent on the number of patients considered, and the error of the predicted rate becomes unacceptable if the numbers are small.

Clearly, a high survival rate is attainable in the treatment of renal failure by home dialysis. Most of the patients in the series have been treated by this method. The fact that transplantation has apparently not adversely affected the survival rate, despite the failure of several transplants, is due to dialysis therapy being restarted after transplant failure.

In computing survival when both dialysis and transplantation have been used in the same patient dialysis periods have been assumed to be continuous. This is because the response to treatment of a patient who rejects a transplant and is returned to dialysis is comparable with that obtained before transplantation, with the possible exception of patients who have serious side effects from treatment with steroids and immunosuppressive agents. No more than one change of treatment occurred in the transplant patients considered here. Apparently survival is unrelated to social status, since both surviving and deceased patients came from all walks of life.
No systematic reason for the improved survival of men over women was found, but possibly women are less well able to tolerate the stress of the treatment. Firm conclusions cannot be drawn at this point, however, since the number of deaths is small. No difference between the survival rates of men and women was found by Lewis et al. (1970).

Though it is difficult to isolate factors contributing to survival, the role of medical, nursing, dietary, and organizational policies is clear. Technically superior performance in dialysis, dietary therapy, and the control of hypertension are of great importance. Similarly, it is essential that the patient assumes responsibility for dialysis and that direct links with advisers in the hospital are provided should a technical or medical problem arise. The patient definitely seems to appreciate and respond to efforts to restore him to his home and natural environment, particularly if this is done sympathetically but objectively.

The five-year overall survival rate of $80.7 \%$ for combined therapy in this programme is an improvement on the fiveyear survival rate of $57.8 \%$ reported by Lewis et al. (1969) for hospital dialysis alone, and probably home dialysis, with a four-year survival rate of $86.2 \%$, is mainly responsible for that improvement. This survival rate is certainly higher than that anticipated by existing plans for the treatment of renal failure, since it implies that very few of the dialysis population would die. Furthermore the results of a recent survey of the need for dialysis in Northern Ireland (M. McGeown, personal communication) show that 33 patients per million per year between the ages of 10 and 55 require regular dialysis therapy. This requirement is not met by the present programme.

\section{REFERENCES}

Baillod, R. A., Knight, A. H., Crockett, R. E, and Naish, P. F. (1969) In Dialysis and Renal Transplantation. Proceedings of the 6th Conference of the European Dialysis and Transplant Association, ed. D. N. S. Kerr,

p. 65. London, Pitman Medical.
Cutler, S. J., and Ederer, F. (1958). Fournal of Chronic Diseases, 8, 699.

Lewis, E. J., Foster, D. M., de la Puente, J., and Scurlock, C. (1969) Annals of Internal Medicine, 70, 311 .

Moorhead, J. F., Baillod, R. A., and Hopewell, J. P. (1970). Proceedings of the 4th International Congress of Nephrology. In press.

\title{
Malabsorption of Dietary Folate (Pteroylpolyglutamates) in Adult Coeliac Disease and Dermatitis Herpetiformis
}

\author{
A. V. HOFFBRAND, ${ }^{*}$ B.M., M.R.C.P., D.C.P. ; A. P. DOUGLAS, † M.B., M.R.C.P. \\ L. FRY, $\ddagger$ M.D., M.R.C.P. ; J. S. STEWART, $§$ M.B., M.R.C.P.
}

British Medical fournal, 1970, 4, 85-89

Summary : Tests of absorption of folic acid (pteroyl$\checkmark$ glutamic acid) and of dietary folates (pteroylpolyglutamates) were performed in 10 patients with untreated adult coeliac disease, five with dermatitis herpetiformis, and three with nutritional folate deficiency. Absorption of pteroylglutamic acid was impaired in eight patients with coeliac disease and in two with dermatitis herpetiformis. Absorption of pteroylpolyglutamates was impaired in all 10 coeliac patients and in four of the five patients with dermatitis herpetiformis. Absorption of both forms of folate was normal in all three patients with nutritional folate deficiency.

\footnotetext{
* Lecturer, Department of Haematology, Royal Postgraduate Medical School, London W.12.

t Research Fellow, Department of Gastroenterology and M.R.C Malabsorption Group, Royal Postgraduate Medical School, London W.12.

¥ Consultant Dermatologist, St. Mary's Hospital, London W.2. Consultant Physician, West Middlesex Hospital, Isleworth,
Middlesex.
}

Jejunal pteroylpolyglutamate hydrolase ("folate
conjugase"), the enzyme thought to be involved in
hydrolysis of dietary pteroylpolyglutamates during their
absorption, was normal in all patients tested, including 17
with adult coeliac disease, four with dermatitis herpe-
tiformis, and three with nutritional folate deficiency.
assayed microbiologically has been shown to occur in this disease by the demonstration of subnormal folate excretion (Girdwood, 1953) and subnormal rise in serum pteroylglutamic acid (Chanarin et al., 1958; Klipstein, 1966) after oral 